ARTICLE

https://doi.org/10.1038/s41467-019-11937-9

\title{
Liquefaction of water on the surface of anisotropic two-dimensional atomic layered black phosphorus
}

Jinlai Zhao 1,2,3, Jiajie Zhu ${ }^{3}$, Rui Cao ${ }^{1,2}$, Huide Wang ${ }^{2}$, Zhinan Guo², David K. Sang ${ }^{2}$, Jiaoning Tang ${ }^{3}$, Dianyuan Fan², Jianqing $\mathrm{Li}^{1} \&$ Han Zhang (D) $^{2}$

The growth and wetting of water on two-dimensional(2D) materials are important to understand the development of 2D material based electronic, optoelectronic, and nanomechanical devices. Here, we visualize the liquefaction processes of water on the surface of graphene, $\mathrm{MoS}_{2}$ and black phosphorus (BP) via optical microscopy. We show that the shape of the water droplets forming on the surface of BP, which is anisotropic, is elliptical. In contrast, droplets are rounded when they form on the surface of graphene or $\mathrm{MoS}_{2}$, which do not possess orthometric anisotropy. Molecular simulations show that the anisotropic liquefaction process of water on the surface of $\mathrm{BP}$ is attributed to the different binding energies of $\mathrm{H}_{2} \mathrm{O}$ molecules on BP along the armchair and zigzag directions. The results not only reveal the anisotropic nature of water liquefaction on the BP surface but also provide a way for fast and nondestructive determination of the crystalline orientation of BP.

\footnotetext{
${ }^{1}$ Faculty of Information Technology, Macau University of Science and Technology, Avenida Wai Long, Taipa, Macau 999078, PR China. ${ }^{2}$ Shenzhen Engineering Laboratory of Phosphorene and Optoelectronics, International Collaborative Laboratory of 2D Materials for Optoelectronics Science and Technology, Engineering Technology Research Center for 2D Material Information Function Devices and Systems of Guangdong Province, Institute of Microscale Optoelectronics (IMO), Shenzhen University, Shenzhen 518060, PR China. ${ }^{3}$ College of Materials Science and Engineering, Shenzhen Key Laboratory of Polymer Science and Technology, Guangdong Research Center for Interfacial Engineering of Functional Materials, Shenzhen 518060, PR China. Correspondence and requests for materials should be addressed to Z.G. (email: guozhinan@szu.edu.cn) or to H.Z. (email: hzhang@szu.edu.cn)
} 
$\mathrm{B}$ lack phosphorus (BP), one of the most stable allotropes of phosphorus $^{1,2}$, has recently (since 2014) emerged as an important two-dimensional (2D) material ${ }^{3,4}$. BP consists of corrugated planes of phosphorus atoms with extremely strong intralayer bonding and weak interlayer interactions ${ }^{5}$. Remarkably, as a metal-free layered semiconductor, the layer-dependent direct band gap of $\mathrm{BP}$ is tunable from $2 \mathrm{eV}$ for a monolayer to $0.3 \mathrm{eV}$ for the bulk material ${ }^{5,6}$. Furthermore, layered BP possesses extremely high carrier mobility $\left(\sim 200-1000 \mathrm{~cm}^{2} \mathrm{~V}^{-1} \mathrm{~s}^{-1}\right)$ and a moderate on/off ratio $\left(\sim 10^{4}-10^{5}\right)^{7,8}$, holding extensive applications in electronic and optoelectronic devices ${ }^{9}$, such as field-effect transistors (FETs) $3,10,11$, photodetectors ${ }^{12}$, and photothermal agents ${ }^{13-15}$. In contrast to graphene and $\mathrm{MoS}_{2}, \mathrm{BP}$ is a 2D material that possesses in-plane anisotropy due to its special intrinsic crystallographic structure caused by its differing bond angles and bond lengths along its orthogonal in-plane directions. Many anisotropic behaviors of BP, including the optical, vibrational, electronic, thermal, and mechanical aspects $1,2,9,16-22$, have been studied. Such anisotropic properties of BP play a vital role in designing polyfunctional and controllable $2 \mathrm{D}$ innovative electronic, optoelectronic, and nanomechanical devices, which are impossible for other isotropic 2D layered materials ${ }^{23}$.

Many experiments and theoretical calculations have been carried out to study the anisotropic properties, and methods have been developed to identify the specific armchair (AC) and zigzag (ZZ) crystalline directions of BP. The electrical and thermal conductance values of layered BP exhibit strong spatial anisotropies. Their respective preferred directions of conductance are mutually orthogonal, leading to an anisotropic electrical $3,17,24$ and thermoelectric $2,9,25$ figure of merit, which is larger along the AC direction. The intrinsic anisotropic light-matter interactions in $\mathrm{BP}$, including the electron-photon interactions, make anisotropic optical absorption and scattering spectroscopy a reliable and simple in situ way to identify the crystalline orientation of $\mathrm{BP}^{21}$. By comparing the ratio of the intensity of the Raman peaks, angle-resolved polarized Raman spectroscopy has become an accurate method to identify the specific $\mathrm{ZZ}$ and AC crystalline directions of $\mathrm{BP}^{19,24}$. In addition to optical methods, mechanical methods can also be applied to distinguish the direction of BP, because the values of the Young's modulus, breaking stress, and elastic modulus of $\mathrm{BP}$ are all higher in the $\mathrm{ZZ}$ direction than in the AC direction ${ }^{22,26}$. However, these methods either rely on expensive equipment, sophisticated data collection and analysis, complex experimental design, or cause some damage to the structure of BP. In this case, a simpler method should be developed for fast in situ identification of the orientation of BP, which does not rely on expensive and complicated equipment.

The growth and motion of atoms, molecules, and clusters on a crystal surface is always an important research topic in the field of surfaces and interfaces. After 2D materials gained popularity since the discovery of graphene, studies on the behaviors of water wetting and diffusion on or in atomic structured layers became possible. Recently, a series of theoretical simulations regarding the behaviors of water on graphene $\mathrm{e}^{27-29}$, boron nitride $\mathrm{e}^{30-32}$, and $\mathrm{MoS}_{2}{ }^{33-35}$ were published, and a mechanism for the surface diffusion of water on these materials was determined. However, experimental results on this topic are rarely reported, especially for atomic layered materials with anisotropic properties, even though the anisotropic wetting characteristics of water droplets on black phosphorene has already been theoretically predicted $^{36,37}$.

Herein, the liquefaction process of water on the surface of $\mathrm{BP}$, which is a typical atomic layer with anisotropic properties, has been first visualized by a microscopy. It has been observed that the shape of the water droplets forming on the surface of $\mathrm{BP}$ is mainly elliptic rather than rounded, as they form on the surfaces of materials not possessing orthometric anisotropy, including graphene and $\mathrm{MoS}_{2}$. The anisotropic liquefaction process of water on the surface of $\mathrm{BP}$ is attributed to the differing binding energies of $\mathrm{H}_{2} \mathrm{O}$ molecules on $\mathrm{BP}$ along the $\mathrm{AC}$ and $\mathrm{ZZ}$ directions. The results not only disclose the anisotropic nature of water liquefaction on the BP surface but also provide a way for fast and nondestructive determination of the crystalline orientation of BP, which is important for future research on anisotropic BP-based electronic, optoelectronic, and nanomechanical devices.

\section{Results}

Liquefaction of water on BP, graphene, and $\mathrm{MoS}_{2}$. To investigate the liquefaction process of water on the surface of BP, a vapor generator and an optical microscope were used ${ }^{38}$, as shown in Supplementary Fig. 1. When the gas phase water coming out from the generator reaches the surface of the $\mathrm{Si} / \mathrm{SiO}_{2}$ wafer carrying $\mathrm{BP}$ flakes, a phase change will happen there (gas phase water changes into the liquid phase). Small droplets will grow large and aggregate as time goes on. When the size of the droplets is large enough, they can be observed by a microscope. Both images and videos of the liquefaction progress of the vapor on the surface of BP flakes were recorded by an optical microscope with
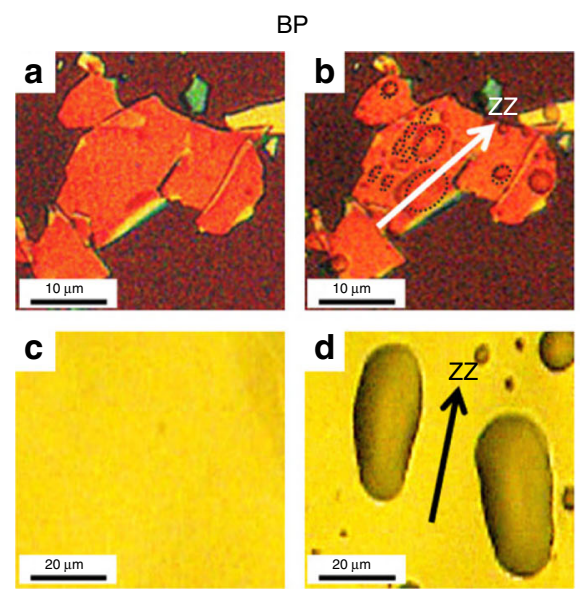
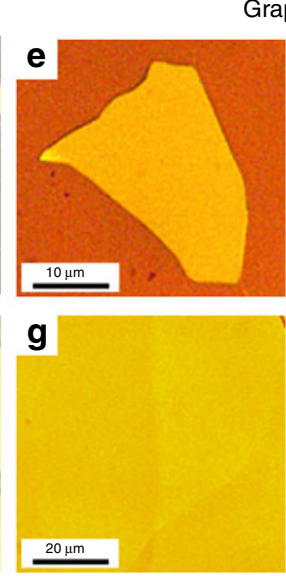

Graphene
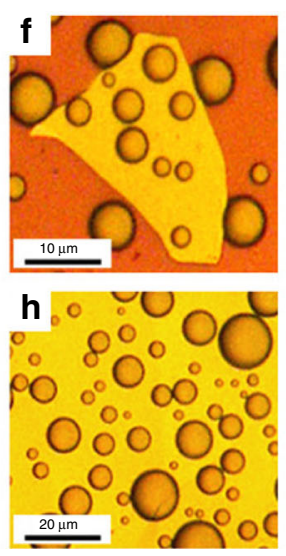

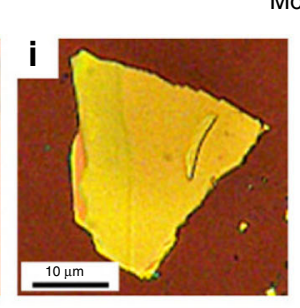

$\mathrm{MoS}_{2}$

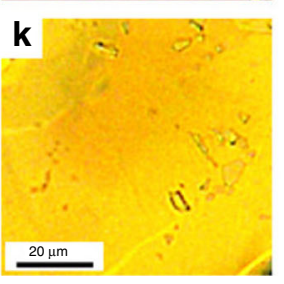

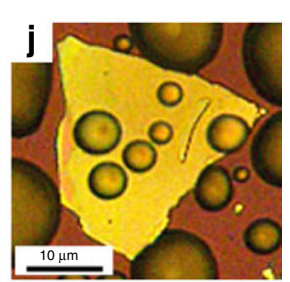

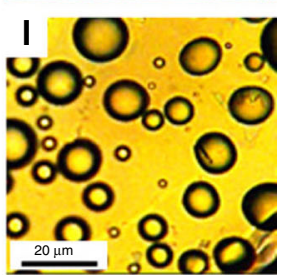

Fig. 1 Optical microscope images of BP/graphene/MoS 2 without and with water droplets. a, e, i Few-layer BP/graphite/MoS 2 without water droplets, $\mathbf{b}, \mathbf{f}, \mathbf{j}$ few-layer BP/graphite/MoS 2 with water droplets, $\mathbf{c}, \mathbf{g}$, $\mathbf{k}$ thick BP/graphite/MoS 2 without water droplets, and $\mathbf{d}, \mathbf{h}, \mathbf{I}$ thick BP/graphite/MoS 2 with water droplets 
$2 \mathrm{~K}$ magnification, as shown in Fig. 1 and Supplementary Movie 1.

Supplementary Movie 1 shows that water vapors first find the condensation nucleus on the BP surface. Then, the condensation progress happens around the nucleus, which leads to very tiny water droplets forming, and the tiny water droplets will become bigger and bigger as time goes on. Statistics, as shown in Supplementary Fig. 2, have been conducted at 12, 13, 14, and $15 \mathrm{~s}$ in Supplementary Movie 1 to demonstrate the shape evolution of water droplets during the growth process. It demonstrates that the water droplets on the surface of BP layer are either elliptical or spherical, but ellipsoids are dominant. In addition, as is shown in Supplementary Fig. 2e, the ratios of long axis and short axis for the elliptical water droplets range from 1.2 to 3.0 , mainly 1.8 as time goes on. Importantly, all long axes of the water droplets point in almost the same direction, as shown in Fig. 1b, $d$.

However, such anisotropic condensation progress of water vapors can only be observed on the surface of anisotropic BP. On an isotropic surface, such as graphite and $\mathrm{MoS}_{2}$, the anisotropic shape of water droplets cannot be observed, as shown in Fig. 1. In contrast, the water droplets on graphene and $\mathrm{MoS}_{2}$ nanosheets are totally round. In addition, Supplementary Movies 2 and 3 show that throughout the whole condensation progress, the water droplets on the surface of graphene and $\mathrm{MoS}_{2}$ are all round rather than elliptical. In summary, the elliptic water droplets can only be observed on the anisotropic surface. In addition, the phenomenon of forming elliptic water droplets on the anisotropic atomic stacked structure surface has nothing to do with the atomic layer thickness of the sample. Figure 1c, d shows that the elliptic water droplets can also be formed on the thick BP surface, but the water droplets on thick graphite and $\mathrm{MoS}_{2}$ sheets remain round (see Fig. 1h,1l).

Direction of elliptic water droplets on BP. To determine the relationship between the distribution directions of the water droplets on the surface of BP sheets and the anisotropic atomic structure of BP, a polarized Raman experiment has been conducted according to the method reported previously 9,19 . The peak positions of all three typical vibrational modes $\left(A_{\mathrm{g}}{ }^{1}, B_{2 \mathrm{~g}}\right.$, and $\left.A_{\mathrm{g}}{ }^{2}\right)$ did not change as the excitation laser polarization angle varied from $0^{\circ}$ to $180^{\circ}$, as shown Fig. 2a. However, all the intensities of the $A_{\mathrm{g}}{ }^{1}, B_{2 \mathrm{~g}}$, and $A_{\mathrm{g}}{ }^{2}$ modes vary periodically with the incident laser polarization changing from $0^{\circ}$ to $180^{\circ}$, which shows obvious angle-dependent anisotropy for BP sheets and is highly consistent with the previous reports $19,20,24$. It has been demonstrated that the relatively larger local maximum intensities of $A_{\mathrm{g}}{ }^{1}$ mode peaks corresponds to the $\mathrm{AC}$ directions of $\mathrm{BP}$, while the relatively

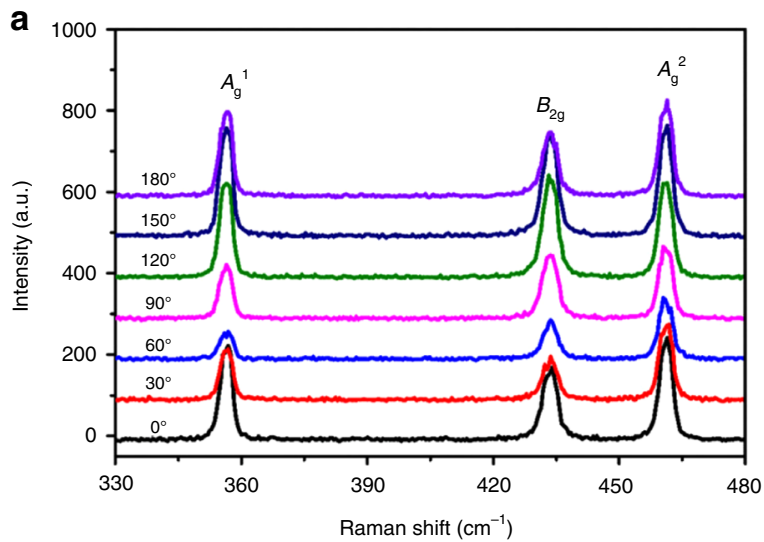

smaller local maximum associates with the $\mathrm{ZZ}$ directions ${ }^{19}$. In this case, a polar diagram based on the intensities of the $A_{\mathrm{g}}{ }^{1}$ mode could be obtained by tuning the angle of the polarization of the incident laser. Based on the polar diagram shown in Fig. 2b, the $\mathrm{AC}$ and $\mathrm{ZZ}$ directions could be determined. By using this polarized Raman method, the long axis of the water droplets has been proven to be along the $\mathrm{ZZ}$ direction. To further confirm this consistency, the polarized Raman measurements and the liquefaction of water experiments were repeated ten times. This finding shows that the difference of the direction of the elliptic water droplets and $\mathrm{ZZ}$ direction of $\mathrm{BP}$ is $<10^{\circ}$. In this case, compared to the angle-resolved polarized Raman spectroscopy, which relies on expensive equipment and complex data collection and analysis, observation of the liquefaction of water on BP could be a much cheaper, faster, and more convenient method to identify the $\mathrm{AC}$ and $\mathrm{ZZ}$ directions of BP sheets.

Mechanism and simulations for anisotropic wetting on BP. One of the physical factors of anisotropic wetting is ascribed to the liquid contact line encountering physical discontinuities such as the sharp solid edges. For example, nanometer- and micrometer-scale surface structures (groves, parallel lines, pillars, wrinkles) can lead to macroscopic, visible changes in anisotropic wetting behaviors ${ }^{39}$. Herein, the anisotropic wetting phenomena of BP could be explained by the anisotropic structure of phosphorene. It is well known that the arrangement of BP atoms makes the BP layer a puckered and anisotropic structure, as the schematic diagram of BP shows in Fig. 3, which graphene and $\mathrm{MoS}_{2}$ do not exhibit. Because the water droplets are suspended on the puckered structure and do not directly contact the bottom of the BP atomic substrate, based on the lattice spacing of BP and the size of each $\mathrm{H}_{2} \mathrm{O}$ molecule, the Cassie model has been used to explain the anisotropic wetting behavior of water ${ }^{40}$. The puckered structure of BP exhibits a larger pinning effect of water drops along the $\mathrm{AC}$ direction than along the $\mathrm{ZZ}$ direction for water liquefaction. The larger pinning effect along the AC direction results in a larger restriction for both wetting and water diffusion. Therefore, the energy barrier for diffusion of water along the AC direction is much larger than that along the $\mathrm{ZZ}$ direction ${ }^{41}$. It is obvious that the water droplets move faster along the $\mathrm{ZZ}$ direction than along the AC direction when the water vapors condense on the surface of BP, which leads to the water droplets forming ellipses on the BP surface. Importantly, the directions of the long axes of the elliptical water droplets are parallel to the $\mathrm{ZZ}$ direction of BP, as shown in Fig. 3b.

From an absorption energy perspective regarding the anisotropic wetting on layered $\mathrm{BP}, \mathrm{ab}$ initio electronic structure

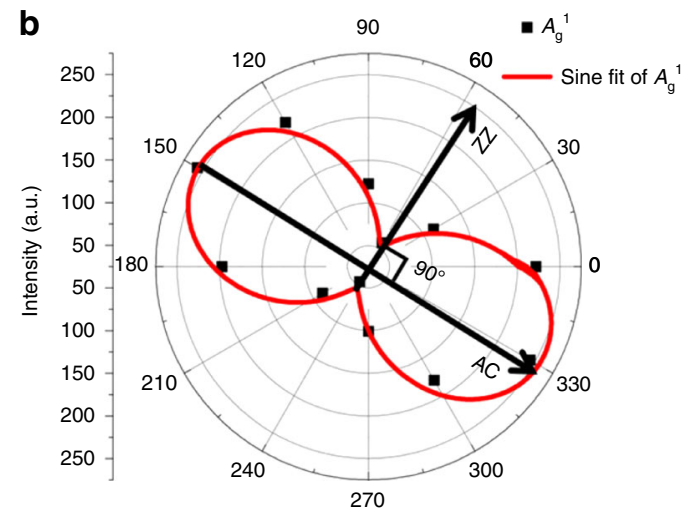

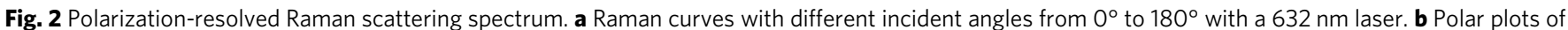
the fitted peak intensities of the $A_{g}{ }^{1}$ modes as a function of sample rotation angle under parallel cross-polarization configurations 
calculations and molecular dynamics simulations were conducted. The calculation results show that the binding energy of $\mathrm{H}_{2} \mathrm{O}$ molecules on phosphorene is $0.17 \mathrm{eV}$, reflecting a weak interaction, which leads to a large vertical distance of $3 \mathrm{~A}$ between

a Side view

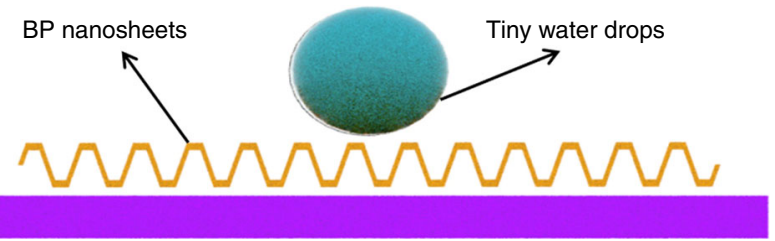

b

Top view

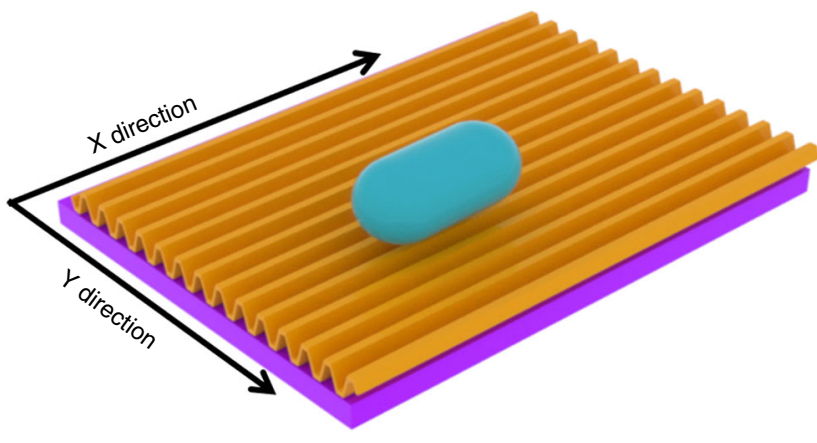

Fig. 3 The schematic diagram of water droplet on the surface of BP. a Side view and $\mathbf{b}$ top view. The $X$ direction is in the zigzag structure and the $Y$ direction is in the armchair structure the molecule and the sheet, which is in agreement with the previous simulation results ${ }^{37}$. The $\mathrm{H}_{2} \mathrm{O}$ molecule almost retains the original structure. In addition, six configurations are considered for $\mathrm{H}_{2} \mathrm{O}$ molecules on phosphorene, namely, along the $\mathrm{AC}, \mathrm{ZZ}$, and diagonal directions, as shown in Fig. 4. Configuration $\mathrm{D}$, showing a large distortion, turns out to be the ground state, with an energy difference of $0.098-0.275 \mathrm{eV}$ molecule ${ }^{-1}$ compared to other configurations. Thus, $\mathrm{H}_{2} \mathrm{O}$ molecules are predicted to align along the $\mathrm{ZZ}$ direction on phosphorene. The result is in accordance with the previous calculation results ${ }^{36,37}$, proving that the energy barrier for diffusion of the water droplets along the AC direction is larger than that along the $\mathrm{ZZ}$ direction for BP layers, which leads to anisotropic diffusion of water droplets. The anisotropic diffusion of water droplets results in anisotropic shape of water droplets, which normally are elliptical on the surface of BP layer just like the optical microscope images showing in the experiment. Moreover, the same simulations of $\mathrm{H}_{2} \mathrm{O}$ molecules on graphene and $\mathrm{MoS}_{2}$ have been carried out as well. The results demonstrate that both graphene and $\mathrm{MoS}_{2}$ possess a ground state among the six configurations for $\mathrm{H}_{2} \mathrm{O}$ adsorption, with an energy difference of $0.008-0.063 \mathrm{eV}$ molecule ${ }^{-1}$ for graphene and $0.003-0.051 \mathrm{eV}$ molecule ${ }^{-1}$ for $\mathrm{MoS}_{2}$, as shown in Supplementary Figs. 4 and 5. However, compared with BP, the energy differences of graphene and $\mathrm{MoS}_{2}$ are much smaller, which leads to inconspicuous anisotropy, and is consistent with the earlier experiment results.

Nondestructive liquefaction of water on BP. As we know that degradation occurs when BP layers are exposed to oxygen and water in air ${ }^{5,42-45}$, so all the wetting experiments were conducted in a glove box with the protection of an inert gas (Ar), as shown in Supplementary Fig. 1. Meanwhile, the BP layers can retain a

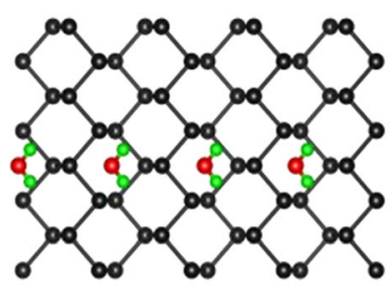

$\infty \quad \infty \quad \infty \quad \infty$

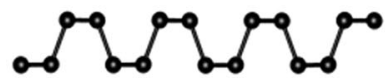

d
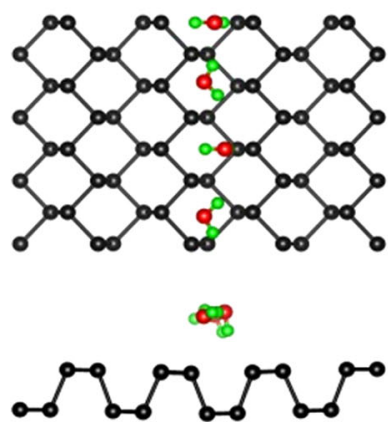

b

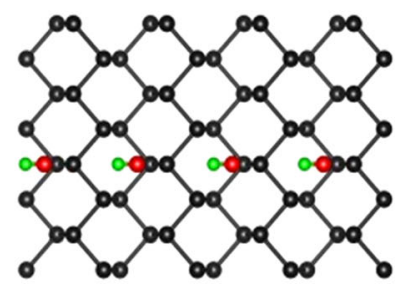

$\%$

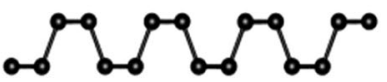

e
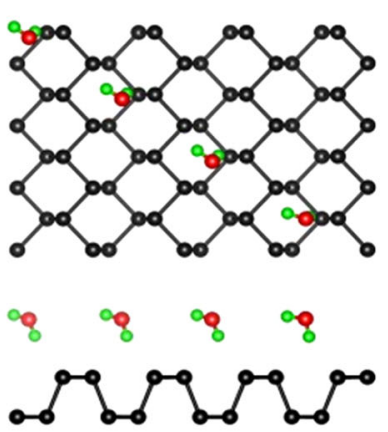

C

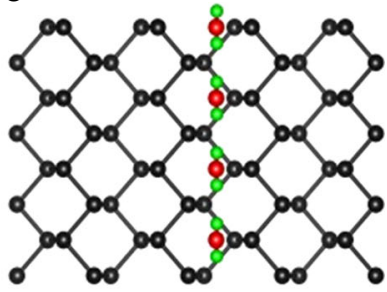

8

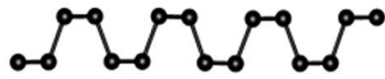

f

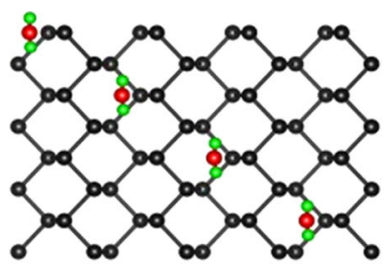

8888

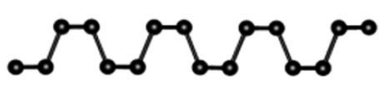

Fig. 4 Six representative high-symmetry configurations for $\mathrm{H}_{2} \mathrm{O}$ molecules on phosphorene. $\mathbf{a}, \mathbf{b}$ Along armchair direction, c, $\mathbf{d}$ along zigzag direction, and

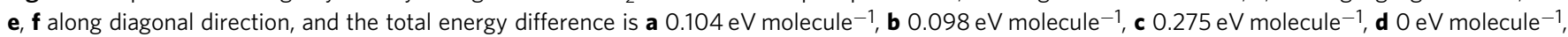
e $0.123 \mathrm{eV}$ molecule $^{-1}$, and $\mathbf{f} 0.150 \mathrm{eV}$ molecule ${ }^{-1}$, respectively 
a

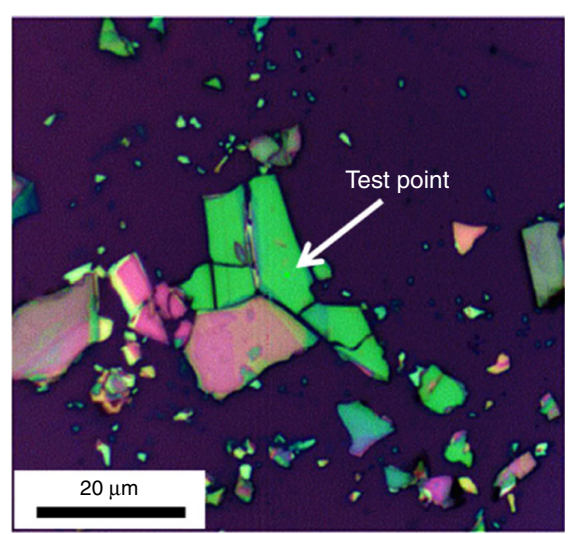

C

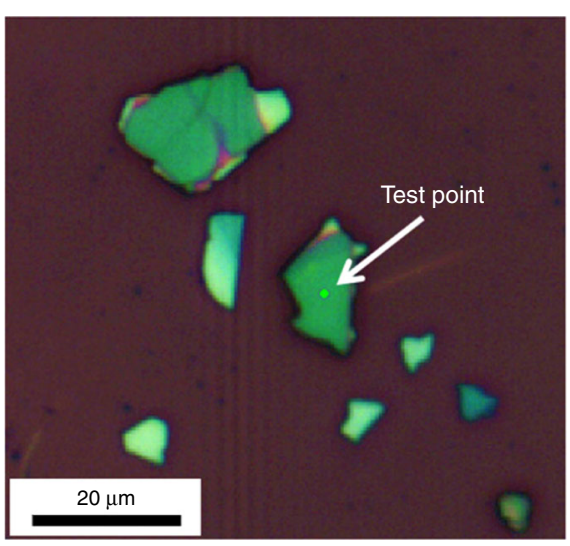

b
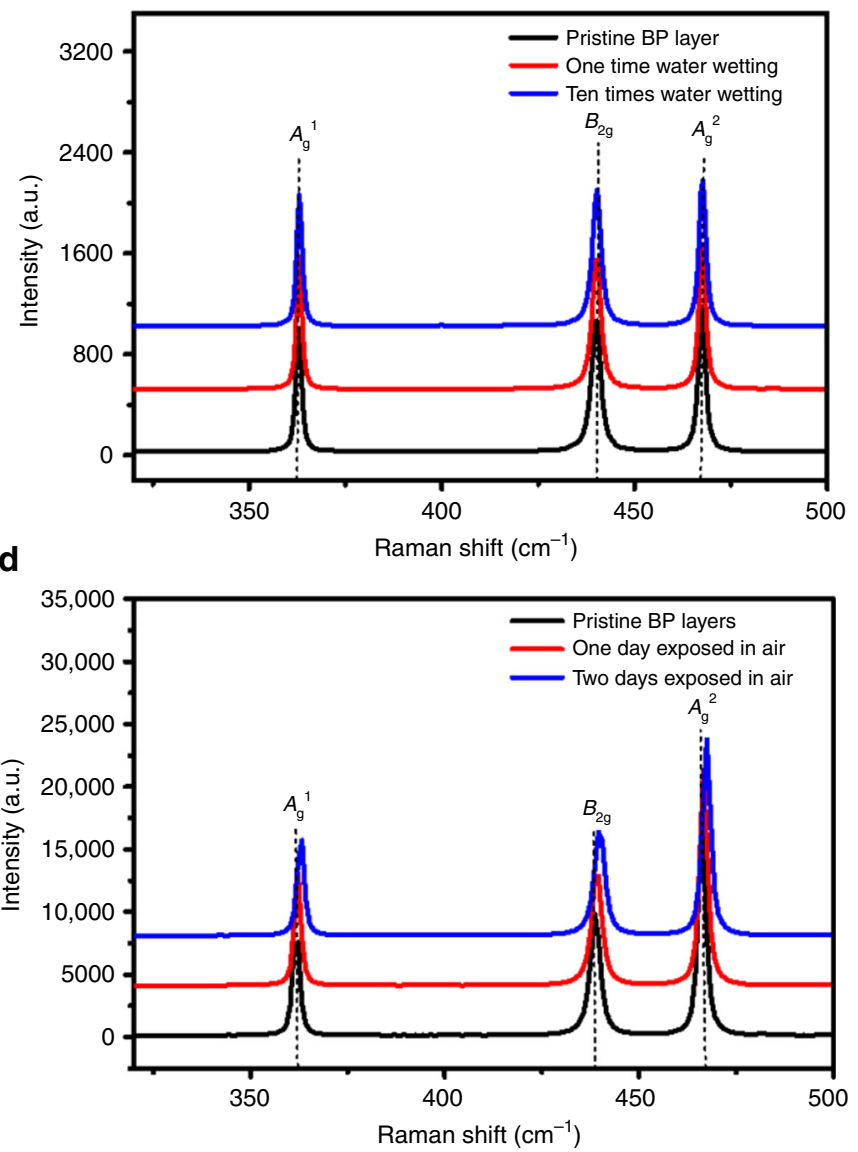

Fig. 5 In situ Raman spectroscopy test of few-layer BP. a, c Optical microscope image of BP layers, b Raman curves of BP nanosheets: pristine (black), after one water wetting experiment (red), and after ten water wetting experiments (blue), and $\mathbf{d}$ Raman curves for pristine BP layers and BP layers 1 and 2 days after exposure to air

their intrinsic properties commendably during the wetting test. As shown in the AFM images of Supplementary Fig. 3, the surfaces of the BP layers almost remain the same without any bubbles after ten wetting experiments. The zoomed AFM image in the yellow dashed line frame (Supplementary Fig. 3c) shows that the Ra can reach $0.22 \mathrm{~nm}$, which is another proof that there was no oxidation reaction on the BP surface. To further demonstrate the nondestructive performance of BP layers in the course of the wetting test, Raman spectroscopy tests in situ for pristine BP layer and for samples after one water wetting experiment and ten water wetting experiments were carried out, as shown in Fig. 5c. All the Raman peak positions and peak intensities of the $A_{\mathrm{g}}{ }^{1}, B_{2 \mathrm{~g}}$, and $A_{\mathrm{g}}{ }^{2}$ peaks at the same test point are almost not changed, which clearly indicates the stable and nondestructive property of BP layers after ten water wetting experiments. In contrast, the Raman curves of pristine BP layers and BP layers 1 and 2 days after exposure to air, as shown in Fig. 5d, clearly demonstrate that all three peaks at $\sim 363,440$, and $466 \mathrm{~cm}^{-1}$, corresponding to the $A_{\mathrm{g}}{ }^{1}, B_{2 \mathrm{~g}}$, and $A_{\mathrm{g}}{ }^{2}$ Raman modes of $\mathrm{BP}$, respectively, move a little to the right, and all the peak intensities decrease. Importantly, the AFM images of the surface of BP layers 1 and 2 days after exposure to air have many bubbles, as shown in Supplementary Fig. 3d, e. Both the Raman and AFM imaging data obviously show the degradation of BP layers ${ }^{43}$ in air for a period of time. Furthermore, a few-layer BP-based FET device $(\sim 8.34 \mathrm{~nm})$ was fabricated; as shown in the inset of Supplementary Fig. $6 \mathrm{~b}$, the transport performance has been tested before and after water liquefaction experiment ${ }^{46}$. The result shows that the $I_{\mathrm{d}}-V_{\mathrm{g}}$ curves almost remain the same before (black line) and after ten wetting experiments (red line), which also demonstrates that there was no structural collapse existing on the BP after ten wetting experiments. In summary, this certainly confirms that the method to identify the anisotropy of BP sheets by using the wetting test is completely nondestructive. It only takes about $10 \mathrm{~s}$ to identify the $\mathrm{BP}$ atomic orientation without destroying the intrinsic atomic structure of $\mathrm{BP}$, which is very important for the application of anisotropic BP-based electronic, optoelectronic, and nanomechanical devices.

\section{Discussion}

In summary, the liquefaction process of water vapors on the surface of $2 \mathrm{D}$ materials was observed via optical microscope. The shapes of the water droplets on the surface of layered BP, possessing anisotropic atomic arrangement, are totally elliptical through all the condensing progress. On the contrary, the shape of the water droplets is apparently round on the isotropic atomic arrangement surface, such as graphene and layered $\mathrm{MoS}_{2}$. One of the physical factors of anisotropic wetting is ascribed to the pinning effect of the water droplets flowing on the BP surface possesses larger energy barrier along the AC direction than along the $\mathrm{ZZ}$ direction. From the perspective of absorption energy, the arrangement of water along the $\mathrm{ZZ}$ direction of $\mathrm{BP}$ needs the smallest binding energy. The method to study the liquefaction process of water vapors on BP surface can be used for fast identification of the orientation of BP structure. Without any 
large-scale instruments and complicated data analyses, the accurate orientation information of BP can be obtained within 10 s. Moreover, this method can be promoted to some other 2D materials possessing both in-plane and out-of-plane anisotropic properties. The study paves a way for future research on anisotropic 2D material-based electronic, optoelectronic, and nanomechanical devices.

\section{Methods}

Sample preparation. All 2D material flakes (BP, graphene, and $\mathrm{MoS}_{2}$ ) were produced from their bulk crystals by a scotch tape-based mechanical exfoliation method and then transferred onto a $\mathrm{Si} / \mathrm{SiO}_{2}(300 \mathrm{~nm})$ wafer by a polydimethylsiloxane film as the medium. During the experiments, all 2D material flakes were characterized immediately after they had been prepared to ensure that all obtained properties are intrinsic, without any structure degradation and contaminations. Additionally, all the few-layered materials studied here were in 8-10 $\mathrm{nm}$ thickness, because $2 \mathrm{D}$ layered materials in such thickness had been most extensively studied and possess relatively better performance for devices research than monolayer ones, especially for BP layers ${ }^{3,47-50}$.

Water droplets forming on the nanosheets. A vapor generator from the Alibaba company had been applied for vapor generation. After the $\mathrm{SiO}_{2} / \mathrm{Si}$ substrates with different kinds of 2D material flakes were fixed on a flat board, the outlet of the generator facing the sample was turned on. The output vapor liquefied on the surface of the 2D material flakes, and microscale droplets formed there. By using an optical microscope, the size and shape of the droplets could be clearly observed. The whole growing process of each droplet was observed and recorded. To prevent the reaction between the BP samples and the oxygen in the air, which would influence the anisotropic property of BP, the whole process was carried out in a glove box under the protection of an argon atmosphere.

Polarization-dependent Raman characterization. Polarized Raman scattering spectroscopy was performed with a homemade Raman system. A polarization analyzer was put between an edge filter and the detector to achieve parallel polarization, while the cross-polarization configuration was obtained by putting a half-wave plate in the incident laser path. Then, polarized Raman spectra with different angles, $\alpha$, were achieved by rotating the half-wave plate with $\alpha / 2$ from the parallel polarization configuration ${ }^{19}$

Computational method. The total energy calculations were performed in the framework of density functional theory using the projector-augmented wave method as implemented in the Vienna Ab initio Simulation Package ${ }^{51}$. The generalized gradient approximation of Perdew, Burke, and Ernzerhof was selected for the exchange-correlation potential ${ }^{52}$. The long-term van der Waals interaction was taken into account by the DFT-D3 approach ${ }^{53}$. The cut-off energy for plane-wave basis sets was set to $500 \mathrm{eV}$. A $4 \times 4 \times 1$ supercell was used to model $\mathrm{H}_{2} \mathrm{O}$ molecule adsorption on phosphorene. A $2 \times 1 \times 1 k$-mesh was used for the Brillouin zone integrations. The structures were relaxed until the residual forces on the atoms declined to $<0.01 \mathrm{eV} \mathrm{A}^{-1}$.

\section{Data availability}

All data that support the findings of this study are available from the corresponding author upon reasonable request.

Received: 2 April 2019 Accepted: 13 August 2019

Published online: 06 September 2019

\section{References}

1. Xia, F. et al. Rediscovering black phosphorus as an anisotropic layered material for optoelectronics and electronics. Nat. Commun. 5, 1-6 (2014).

2. Fei, R. et al. Enhanced thermoelectric efficiency via orthogonal electrical and thermal conductances in phosphorene. Nano Lett. 14, 6393-6399 (2014).

3. Li, L. et al. Black phosphorus field-effect transistors. Nat. Nanotechnol. $\mathbf{9}$, 372-377 (2014).

4. Du, Y. et al. Device perspective for black phosphorus field-effect transistors: Contact resistance, ambipolar behavior, and scaling. ACS Nano. 8 , 10035-10042 (2014).

5. Ryder, C. R. et al. Covalent functionalization and passivation of exfoliated black phosphorus via aryl diazonium chemistry. Nat. Chem. 8, 597-602 (2016).

6. Zhao, Y. et al. Surface coordination of black phosphorus for robust air and water stability. Angew. Chem. Int. Ed. Engl. 55, 5003-5007 (2016).
7. Guo, Z. et al. Metal-ion-modified black phosphorus with enhanced stability and transistor performance. Adv. Mater. 29, 1-8 (2017).

8. Han, C. et al. Surface functionalization of black phosphorus via potassium toward high-performance complementary devices. Nano Lett. 17, 4122-4129 (2017).

9. Luo, Z. et al. Anisotropic in-plane thermal conductivity observed in few-layer black phosphorus. Nat. Commun. 6, 1-8 (2015).

10. Buscema, M. et al. Fast and broadband photoresponse of few-layer black phosphorus field-effect transistors. Nano Lett. 14, 3347-3352 (2014).

11. Na, J. et al. Few-Layer black phosphorus field-effect transistors with reduced current fluctuation. ACS Nano 8, 11753-11762 (2014).

12. Ren, X. et al. Environmentally robust black phosphorus nanosheets in solution: application for self-powered photodetector. Adv. Funct. Mater. 27, 1-8 (2017).

13. Sun, Z. et al. Ultrasmall black phosphorus quantum dots: synthesis and use as photothermal agents. Angew. Chem. Int. Ed. Engl. 54, 11526-11530 (2015).

14. Shao, J. et al. Biodegradable black phosphorus-based nanospheres for in vivo photothermal cancer therapy. Nat. Commun. 7, 1-13 (2016).

15. Chen, W. et al. Black phosphorus nanosheet-based drug delivery system for synergistic photodynamic/photothermal/chemotherapy of cancer. Adv. Mater. 29, 1-7 (2017).

16. Fei, R. et al. Strain-engineering the anisotropic electrical conductance of fewlayer black phosphorus. Nano Lett. 14, 2884-2889 (2014).

17. Qiao, J. et al. High-mobility transport anisotropy and linear dichroism in fewlayer black phosphorus. Nat. Commun. 5, 1-7 (2014)

18. Wang, X. et al. Highly anisotropic and robust excitons in monolayer black phosphorus. Nat. Nanotechnol. 10, 517-521 (2015).

19. Wu, J. et al. Identifying the crystalline orientation of black phosphorus using angle-resolved polarized Raman spectroscopy. Angew. Chem. Int. Ed. Engl. 54 2366-2369 (2015)

20. Yuan, H. et al. Polarization-sensitive broadband photodetector using a black phosphorus vertical p-n junction. Nat. Nanotechnol. 10, 707-713 (2015).

21. Ling, X. et al. Anisotropic electron-photon and electron-phonon interactions in black phosphorus. Nano Lett. 16, 2260-2267 (2016).

22. Wang, Z. et al. Resolving and tuning mechanical anisotropy in black phosphorus via nanomechanical multimode resonance spectromicroscopy. Nano Lett. 16, 5394-5400 (2016).

23. Jiang, H. et al. Optical anisotropy of few-layer black phosphorus visualized by scanning polarization modulation microscopy. ACS Photonics 5, 2509-2515 (2018).

24. Liu, X. et al. Resolving the in-plane anisotropic properties of black phosphorus. Small Methods 1, 1-9 (2017).

25. Sun, B. et al. Temperature dependence of anisotropic thermal-conductivity tensor of bulk black phosphorus. Adv. Mater. 29, 1-8 (2017).

26. Chen, H. et al. Anisotropic mechanical properties of black phosphorus nanoribbons. J. Phys. Chem. 120, 29491-29497 (2016).

27. Nair, R. R. et al. Unimpeded permeation of water through helium-leak-tight graphene-based membranes. Science 335, 442-444 (2012).

28. Rafiee, J. et al. Wetting transparency of graphene. Nat. Mater. 11, 217-222 (2012).

29. Ma, M. et al. Fast diffusion of water nanodroplets on graphene. Nat. Mater. 15, 66-71 (2016).

30. $\mathrm{Li}, \mathrm{H}$. et al. Wetting and interfacial properties of water nanodroplets in contact with graphene and monolayer boron-nitride sheets. ACS Nano 6, 2401-2409 (2012).

31. Lei, W. et al. Porous boron nitride nanosheets for effective water cleaning. Nat. Commun. 4, 1-7 (2013).

32. Liu, F. et al. Nanosheet-structured boron nitride spheres with a versatile adsorption capacity for water cleaning. ACS Appl. Mater. Interfaces 7 , 1824-1832 (2015).

33. Chow, P. K. et al. Wetting of mono and few-layered $\mathrm{WS}_{2}$ and $\mathrm{MoS}_{2}$ films supported on $\mathrm{Si} / \mathrm{SiO}_{2}$ substrates. ACS Nano 9, 3023-3031 (2015).

34. Li, W. et al. Tunable, strain-controlled nanoporous $\mathrm{MoS}_{2}$ filter for water desalination. ACS Nano. 10, 1829-1835 (2016).

35. Luan, B. et al. Wettability and friction of water on a $\mathrm{MoS}_{2}$ nanosheet. Appl. Phys. Lett. 108, 1-5 (2016).

36. Zhang, W. et al. Molecular structure and dynamics of water on pristine and strained phosphorene: wetting and diffusion at nanoscale. Sci. Rep. 6, 1-9 (2016).

37. Chen, S. et al. Anisotropic wetting characteristics of water droplets on phosphorene: roles of layer and defect engineering. J. Phys. Chem. C 122, 4622-4627 (2018).

38. Castellanos-Gomez, A. et al. Isolation and characterization of few-layer black phosphorus. 2D Mater. 1, 1-19 (2014).

39. Xia, D. et al. Anisotropic wetting surfaces with one-dimensional and directional structures: fabrication approaches, wetting properties and potential applications. Adv. Mater. 24, 1287-1302 (2012). 
40. Xia, D. et al. Strongly anisotropic wetting on one-dimensional nanopatterned surfaces. Nano Lett. 8, 2819-2824 (2008).

41. Johnson Jr, R. E. et al. Contact angle hysteresis. III. Study of an idealized heterogeneous surface. J. Phys. Chem. 68, 1744-1750 (1964).

42. Pei, J. et al. Producing air-stable monolayers of phosphorene and their defect engineering. Nat. Commun. 7, 1-8 (2016).

43. Abellan, G. et al. Fundamental insights into the degradation and stabilization of thin layer black phosphorus. J. Am. Chem. Soc. 139, 10432-10440 (2017).

44. Wu, S. et al. Black phosphorus: degradation favors lubrication. Nano Lett. 18, 5618-5627 (2018).

45. Zhang, T. et al. Degradation chemistry and stabilization of exfoliated few-layer black phosphorus in water. J. Am. Chem. Soc. 140, 7561-7567 (2018)

46. Joshua O Island, G. et al. Environmental instability of few-layer black phosphorus. 2D Mater. 2, 1-6 (2015).

47. Liu, H. et al. Semiconducting black phosphorus: synthesis, transport properties and electronic applications. Chem. Soc. Rev. 44, 2732-2743 (2015).

48. Guo, Q. et al. Black phosphorus mid-infrared photodetectors with high gain. Nano Lett. 16, 4648-4655 (2016).

49. Liu, H. et al. Phosphorene: an unexplored 2D semiconductor with a high hole mobility. ACS Nano 8, 4033-4041 (2014).

50. Yuan, H. et al. Polarization-sensitive broadband photodetector using a black phosphorus vertical p-n junction. Nat. Nanotechnol. 10, 707-714 (2015).

51. Kresse, G. et al. From ultrasoft pseudopotentials to the projector augmentedwave method. Phys. Rev. B 59, 1758-1775 (1999).

52. Perdew, J. P. et al. Generalized gradient approximation made simple. Phys. Rev. Lett. 77, 3865-3868 (1996).

53. Grimme, S. et al. A consistent and accurate ab initio parametrization of density functional dispersion correction (DFT-D) for the 94 elements H-Pu. J. Chem. Phys. 132, 1-19 (2010).

\section{Acknowledgements}

We would like to thank Dr. Yani Chen and Prof. Mingyuan Huang from the Department of Physics in the Southern University of Science and Technology (SUST) for their efforts on the polarized Raman measurements. We authors acknowledge financial support from the National Natural Science Fund (Grant Nos. 61605131, 61435010, and 51778369), Guangdong Science Foundation for Distinguished Young Scholars (2018B030306038), Science and Technology Innovation Commission of Shenzhen (Grant Nos.

JCYJ20180507182047316, KQJSCX2018032809550179, KQTD2015032416270385,

JCYJ20150625103619275, and ZDSYS201707271014468), Educational Commission of
Guangdong Province (2016KCXTD006), and the Science and Technology Development Fund (Grant No. 007/2017/A1 and 132/2017/A3), Macao SAR, China.

\section{Author contributions}

J.L.Z., Z.G., and H.Z. conceptualized this work; J.L.Z. developed the methodology; J.L.Z. carried out the water wetting test, Raman measurements, and AFM investigation; J.J.Z. made the simulation calculation; R.C., H.W., and D.K.S. help to process the data; J.L.Z. wrote the original draft; all authors reviewed and edited the manuscript; Z.G. and J.T. acquired funds; H.Z., J.L., and D.F. supervised this work.

\section{Additional information}

Supplementary Information accompanies this paper at https://doi.org/10.1038/s41467019-11937-9.

Competing interests: The authors declare no competing interests.

Reprints and permission information is available online at http://npg.nature.com/ reprintsandpermissions/

Peer Review Information: Nature Communications thanks Gang Zhang and other anonymous reviewer(s) for their contribution to the peer review of this work. Peer reviewer reports are available.

Publisher's note: Springer Nature remains neutral with regard to jurisdictional claims in published maps and institutional affiliations.

Open Access This article is licensed under a Creative Commons Attribution 4.0 International License, which permits use, sharing, adaptation, distribution and reproduction in any medium or format, as long as you give appropriate credit to the original author(s) and the source, provide a link to the Creative Commons license, and indicate if changes were made. The images or other third party material in this article are included in the article's Creative Commons license, unless indicated otherwise in a credit line to the material. If material is not included in the article's Creative Commons license and your intended use is not permitted by statutory regulation or exceeds the permitted use, you will need to obtain permission directly from the copyright holder. To view a copy of this license, visit http://creativecommons.org/ licenses/by/4.0\%

(c) The Author(s) 2019 